Louisiana State University

LSU Digital Commons

$3-1-2020$

\title{
Guiding and Trapping Cracks with Compliant Inclusions for Enhancing Toughness of Brittle Composite Materials
}

\author{
Neal R. Brodnik \\ California Institute of Technology \\ Chun Jen Hsueh \\ California Institute of Technology \\ Katherine T. Faber \\ California Institute of Technology \\ Blaise Bourdin \\ Louisiana State University \\ Guruswami Ravichandran \\ California Institute of Technology
}

See next page for additional authors

Follow this and additional works at: https://digitalcommons.Isu.edu/mathematics_pubs

\section{Recommended Citation}

Brodnik, N., Hsueh, C., Faber, K., Bourdin, B., Ravichandran, G., \& Bhattacharya, K. (2020). Guiding and Trapping Cracks with Compliant Inclusions for Enhancing Toughness of Brittle Composite Materials. Journal of Applied Mechanics, Transactions ASME, 87 (3) https://doi.org/10.1115/1.4045682

This Article is brought to you for free and open access by the Department of Mathematics at LSU Digital Commons. It has been accepted for inclusion in Faculty Publications by an authorized administrator of LSU Digital Commons. For more information, please contact ir@lsu.edu. 


\section{Authors}

Neal R. Brodnik, Chun Jen Hsueh, Katherine T. Faber, Blaise Bourdin, Guruswami Ravichandran, and Kaushik Bhattacharya 
Neal R. Brodnik
Division of Engineering and Applied Science,
California Institute of Technology,
Pasadena, CA
e-mail: nbrodnik@caltech.edu

Chun-Jen Hsueh ${ }^{1}$

Division of Engineering and Applied Science,

California Institute of Technology,

Pasadena, CA

e-mail: renhsueh@gmail.com

Katherine T. Faber

Division of Engineering and Applied Science, California Institute of Technology, Pasadena, CA

e-mail: ktfaber@caltech.edu

Blaise Bourdin

Department of Mathematics, Louisiana State University, Baton Rouge, LA

e-mail: bourdin@|su.edu

\section{Guruswami Ravichandran \\ Division of Engineering and Applied Science, California Institute of Technology, Pasadena, CA \\ e-mail: ravi@caltech.edu}

\author{
Kaushik Bhattacharya \\ Division of Engineering and Applied Science, \\ California Institute of Technology, \\ Pasadena, CA \\ e-mail: bhatta@caltech.edu
}

Guiding and Trapping Cracks With Compliant Inclusions for Enhancing Toughness of Brittle Composite Materials

The problem of toughening heterogeneous materials with a stiff matrix and compliant inclusions is investigated through numerical simulations and experiments. Specifically, the problem of optimizing a combination of effective toughness and effective elastic modulus in the context of a square array of compliant inclusions in a stiff matrix is explored. Crack propagation in the heterogeneous material is simulated using a variational phasefield approach. It is found that the crack can meander between or get attracted to and trapped in the inclusions. Composite specimens with a stiff matrix and compliant circular inclusions were $3 D$ printed, and their fracture toughness was measured using a specially designed loading fixture. The experimental results show agreement with the numerical predictions by demonstrating the attraction and trapping of cracks in the inclusions. This study demonstrates the potential for significant enhancement of toughness through elastic compliance contrast between the matrix and the inclusion without notably compromising the effective elastic modulus of the composite material. [DOI: 10.1115/1.4045682]

Keywords: heterogeneous materials, fracture toughness, phase-field method, additive manufacturing, elastic mismatch

\section{Introduction}

It is understood that the microstructure of a material can have a significant influence on its fracture toughness although the relationship between the two is not always straightforward. In many cases, complexity arises from the introduction of heterogeneous microstructural features that affect failure response differently depending on their shape, size, and chemistry. The toughening benefits of some of these types of features have been explored in different forms in brittle ceramics [1-7]. Heterogeneous structures have also been exploited by nature as a means to improve toughness in materials such as bone and nacre, which has led to numerous bio-inspired composites [8-13].

One of the greatest challenges in developing heterogeneous structures is utilizing the interaction between constituent materials in a beneficial way. If we consider the elastic modulus versus toughness space, as shown in Fig. 1, most materials that demonstrate high toughness also demonstrate high stiffness. However, looking closely at the figure reveals another trend: among brittle materials - polymers and ceramics, the critical energy release rate $G_{c}$ is inversely proportional to stiffness, and it is challenging to find materials that deviate from this general trend. Many composites depart from this trend with high stiffness and critical energy release rate, but this is still limited due to processing constraints that reduce

\footnotetext{
${ }^{1}$ Authors contributed equally to this work.

Contributed by the Applied Mechanics Division of ASME for publication in the Journal OF APPLIED Mechanics. Manuscript received October 31, 2019; final manuscript received November 28, 2019; published online December 12, 2019. Assoc. Editor: Yong-Wei Zhang.
}

both the topological freedom and placement control of microstructural features in heterogeneous systems. This limited control means that studying crack interactions with microstructural features in heterogeneous materials is often restricted to statistical characterizations of bulk composite properties. To this extent, the relationship between random microstructures and observable features has been explored in brittle systems [14-18].

In this study, we look to reach beyond the limitations of traditional composite processing and explore the design space of heterogeneous structures with straightforward methods that can be readily understood and expanded. Here, 3D printing is used to produce controlled and repeatable arrangements of compliant, low-toughness inclusions that can attract and trap cracks within the structure. To mitigate the effect of interfaces, the compliant inclusions were made through reductions in thickness in an otherwise twodimensional composite structure. This ensured perfect material compatibility and also allowed for relative material properties to be precisely tailored through thickness. Using this configuration, we demonstrate that the introduction of inclusions into a homogeneous structure can provide significant toughening to the system even when the inclusions are of lower toughness than the matrix itself, allowing this heterogeneous structure to extend well beyond the traditional rule of mixtures behavior seen in many composite systems. Additionally, we demonstrate that the precise control afforded by 3D printing allows for the fabrication of composite structures with higher toughness at little cost to the stiffness of the structure.

First, we consider how a composite structure with a specific arrangement of circular inclusions might be explored from a numerical sense using a variational phase-field fracture model. We then 


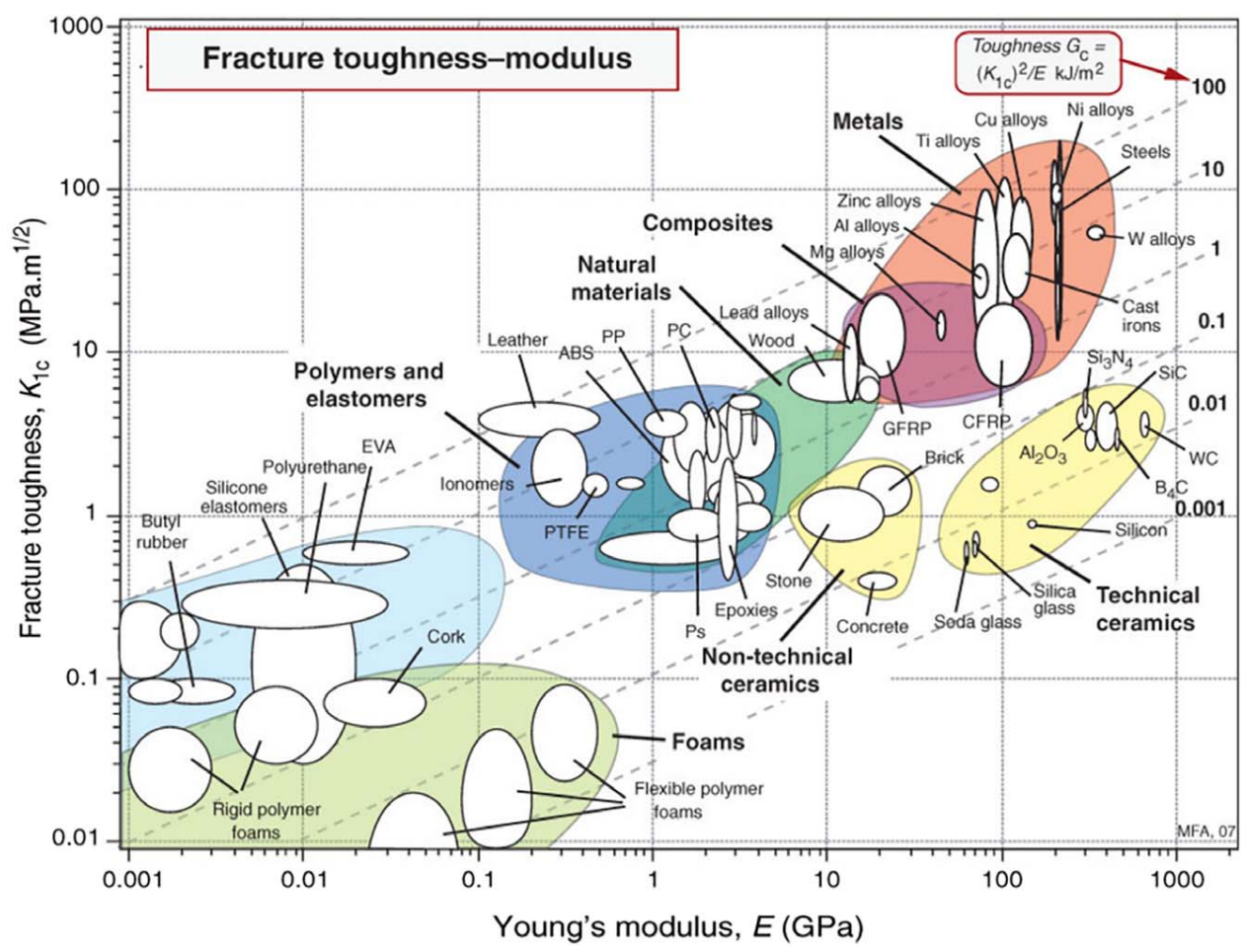

Fig. 1 Materials design space showing the tradeoff between toughness and elastic modulus across material and composite systems. Image created using GRANTA CES EduPack Software from ANSYS Inc. @ 2010.

examine how this same composite design can be produced and characterized with similar material properties using $3 \mathrm{D}$ printing. Some different examples of the phase-field model are used to demonstrate how inclusion properties, namely the stiffness and toughness, can influence the properties of the overall structure when incorporated into the chosen arrangement. Finally, the numerical analyses are compared with experimental results, and increased toughness is demonstrated from the incorporation of inclusions that guide and trap cracks through a combination of compliance contrast and geometric effects.

\section{Computational Approach}

Both the computation and experimental analyses in this work focus on a face-centered-square array of inclusions, as shown in Fig. 2. Simulations are performed using the phase-field fracture method following Bourdin et al. [19-22] to study crack propagation numerically. This is a regularized method that introduces a dimensionless scalar fracture field $\alpha$ taking values in $[0,1]$ to describe the material state. The intact material is represented as $\alpha=0$ and regularized cracks are represented by regions where $\alpha$ localizes with peak value 1 . The computation proceeds by alternately minimizing the functional

$$
\mathcal{E}_{\ell}(u, \alpha)=\int_{\Omega}\left((1-\alpha)^{2}+\eta\right) W(\mathrm{e}(u))+\frac{3 G_{c}}{8}\left(\frac{\alpha}{\ell}+\ell|\nabla \alpha|^{2}\right) d x
$$

over the displacement field $u$ and the fracture field $\alpha$ subject to the constraints $0 \leq \alpha \leq 1$ and $\dot{\alpha} \geq 0$; the latter representing the fact that cracks cannot heal. Above, $W$ is the elastic energy density, $G_{c}$ is the toughness (critical energy release rate), and $\eta$ is a dimensionless small parameter for numerical convenience. The parameter $\ell>0$ is an internal length over which the cracks are regularized, meaning that cracks are represented by regions of width $\mathcal{O}(\ell)$ along the center of which $\alpha=1$. The specific expression of (1) was introduced in Ref. [23] following the analysis in Ref. [24,25] and has been shown [26] to better account to crack nucleation in a variety of settings that the classical models originally introduced in Refs. [20,22]. We use the software vDef [27] for the numerical simulations.

The effective toughness of a heterogeneous material is computed using the surfing boundary method following Hossain et al. [28]. In this method, a large domain (comprising of a large number of unit cells) is considered and subjected to a time-dependent displacement boundary condition corresponding to a translating crack opening displacement,

$$
u^{*}\left(x_{1}, x_{2}, t\right)=U\left(x_{1}-c t, x_{2}\right)
$$

where $U$ is some displacement field that opens the crack, $x_{1}$ is the macroscopic crack propagation direction, $x_{2}$ is the normal to $x_{1}, c$ is the macroscopically prescribed velocity of the boundary condition, and $t$ is the time. In this work, we take the mode-I crack

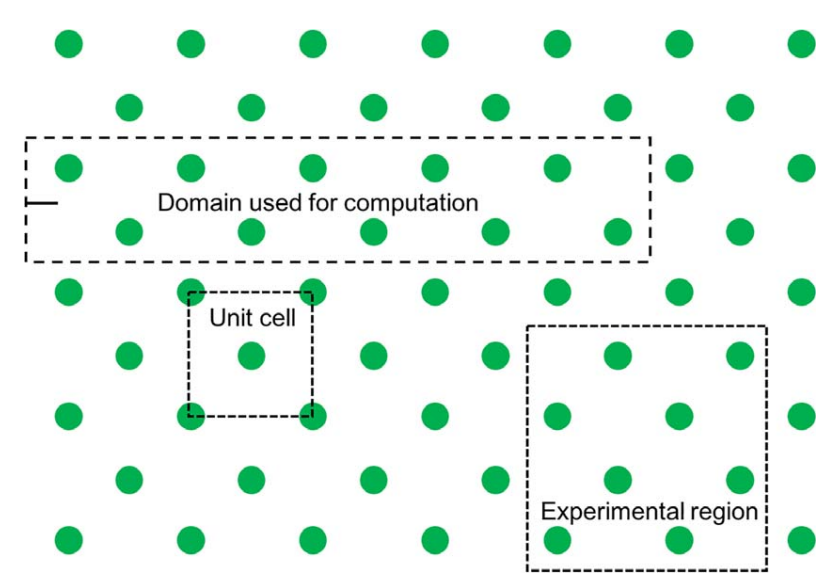

Fig. 2 The face-centered-square array of compliant inclusions in a stiff matrix with the unit cell, the computational domain for fracture and the experimental region marked 
opening displacement

$$
U\left(x_{1}, x_{2}\right)=\frac{K_{I}}{2 \mu} \sqrt{\frac{r}{2 \pi}}(\kappa-\cos \theta)\left(\cos \frac{\theta}{2} \hat{e}_{1}+\sin \frac{\theta}{2} \hat{e}_{2}\right)
$$

where $U$ is the crack opening displacement field, $K_{I}$ is the stress intensity factor, $\mu$ is the shear modulus, $\kappa$ is a function of Poisson's ratio, $r$ is the polar distance to the origin, and $\theta$ is the polar angle. At each time increment, we find the displacement and fracture fields using the method outlined above. Note that the fracture process is unconstrained within the domain - the crack may grow as it chooses (it may deflect, kink, pin, jump) or new cracks may be nucleated depending on the heterogeneity. The driving force at the boundary required to continue the fracture process fluctuates as the process proceeds, and this can be calculated using the $J$-integral on a contour at the boundary of the computational domain. The effective toughness of the material is the peak value of the $J$-integral. This value is independent of the domain, the imposed macroscopic translation velocity, and $U$ as long as the fracture process is confined away from the boundaries.

We study particular composites where circular compliant isotropic inclusions are embedded in a stiff isotropic matrix. We nondimensionalize our parameters so that the toughness $\left(G_{c}\right)$ and elastic (Young's) modulus are unity in the matrix. The effective elastic modulus of the periodic composite containing compliant inclusions is computed on a unit cell using a fast Fourier transform (FFT) method following Moulinec et al. [29-31]. In cases where the inclusion and matrix toughness vary, the interfacial toughness is always equal to the smaller of the values of the two materials separated by the interface. The nucleation length is set as $\ell=0.45$. To establish a crack position, a small pre-crack is inserted into the computational domain as indicated. Further details can be found in Ref. [32].

\section{Experimental Design}

3.1 Composite Design. Designs of traditional brittle materials often use volume fraction when describing the quantity of inclusions, dispersoids, pores, or toughening phases present in a particular composite design. This volume fraction, along with a description of the size and structure of the inclusions as well as the assumption that the inclusion phase is randomly distributed, gives a clear description of heterogeneous structure within the composite. However, because additive manufacturing allows for control of the exact location of heterogeneities, volume fraction is an inadequate descriptor, as there is an infinite number of configurations of inclusions within a bulk structure, both uniform and nonuniform, that can be described by the same bulk volume fraction. This same principle holds with inclusion structure. In conventional composite theory, the influence of both structure and spacing of inclusions on fracture behavior has been explored for the most common inclusion shapes, including rods, spheres, and plates [2].
However, if inclusions can be readily designed to be any shape or structure, this together with spacing make the design space for composites with designed anisotropic structures very large.

To better constrain the design space for the composite structure, we minimized the number of geometric and material parameters while ensuring two-dimensional studies. Previously, layered printed structures were explored in double cantilever beam testing, but all structure and geometry in that study were effectively constrained to one dimension [33]. We chose to investigate arrays of circular inclusions in a face-centered-square array oriented such that the edges of the square are parallel to the unperturbed direction of crack growth, as shown in Fig. 2.

In addition to a fixed arrangement with variable radius, elastic contrast was varied by changing the thickness of inclusion, as shown in Fig. 3(b). This ensured that the effective elastic modulus could be varied without unintended interfacial interactions or material incompatabilities. Furthermore, this allowed the entire composite to be printed from the same photopolymer. The effective elastic modulus of the inclusion $E^{i n c}$ is related to the thickness contrast as follows:

$$
E^{\text {inc }} \propto \frac{t^{\text {inclusion }}}{t^{\text {matrix }}}
$$

where $t^{\text {inclusion }}$ is the inclusion thickness and $t^{\text {matrix }}$ is the matrix material thickness. Note that this is not an actual change in material properties, as the material is the same for both the matrix and inclusions. However, because both thin and thick regions are both subjected to the same far-field loading, the inclusions will experience greater stress for the same loading. Consequently, they will effectively behave as though they have greater compliance than the surrounding matrix. This same principle applies to toughness,

$$
\mathcal{G}_{c}^{\text {inc }}=\frac{\left(K_{\text {Ic }}^{\text {inc }}\right)^{2}}{E^{\text {inc }}} \propto \frac{t^{\text {inclusion }}}{t^{\text {matrix }}}
$$

where $\mathcal{G}_{c}^{\text {inc }}$ is the effective critical energy release rate of the inclusion and $K_{I c}^{i n c}$ is the effective fracture toughness, which, like stiffness, scales with the applied stress. Again, similar to the stiffness, no material properties are actually changing, but because thickness variation changes the stress response to equivalent far-field loading, the relative toughnesses of the inclusion and matrix are different with respect to one another. Once the effective modulus and toughness of the inclusions are determined, these values can be incorporated into the composite design for a given geometry and unit cell arrangement. From here, the effective elastic modulus of the composite $E^{\text {eff }}$ can be determined using FFT, and the effective strain energy release rate of the composite $\mathcal{G}_{c}^{\text {eff }}$ can be determined from the macroscopic $J$-integral [29-31].

3.2 Specimen Fabrication. All samples investigated in this study were fabricated using digital light processing on an Ember

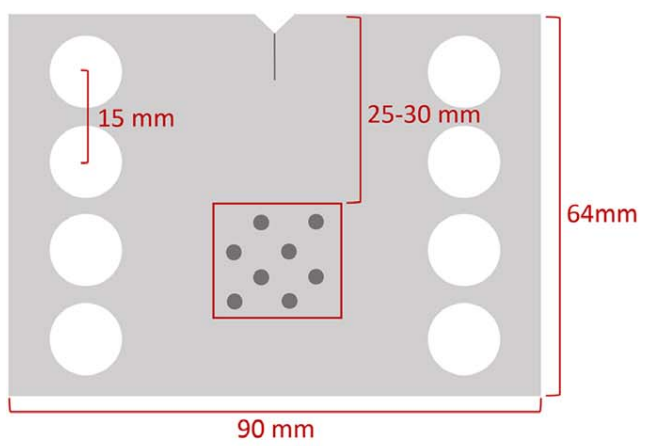

(a)

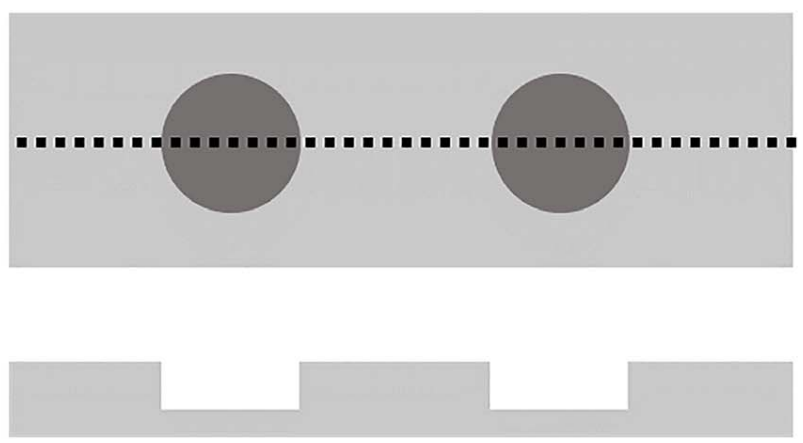

(b)

Fig. 3 Schematic design of (a) a surfing load specimen and (b) the compliant inclusion cross-section 
3D Printer (Autodesk, San Rafael, CA). All were printed using either a clear system, PR48 or an opaque pigmented system, PR57 (Autodesk, San Rafael, CA). Both polymers are urethane acrylate photopolymer blends with compositions reported in Ref. [34]. Both polymers also have similar elastic moduli of about $750 \mathrm{MPa}$ and similar fracture toughnesses of about $0.2 \mathrm{MPa}$ $\sqrt{\mathrm{m}}$. Using either polymer, the Autodesk Ember has a print voxel size of $50 \times 50 \times 50 \mu \mathrm{m}^{3}$ which is well suited to printing surfing load specimens with high fidelity. The design for the surfing load specimens is shown in Fig. 3.

Fracture specimens for surfing load conditions (described below) were printed to be $90 \mathrm{~mm} \times 64 \mathrm{~mm}$ by $2.5 \mathrm{~mm}$ (Fig. 3), with some additional thickness reinforcement present around the pinholes to prevent failure at the loading points. Printed samples, which were $2.5 \mathrm{~mm}$ thick, were sufficiently thin to prevent any unwanted bending moments due to the thickness variation in the inclusions. Inclusion thickness was chosen to be one-fifth of the "bulk" sample thickness. The inclusion and matrix layers were also made flush with one another on one face of the sample, as shown in Fig. $3(b)$ to provide a single planar face for displacement mapping with the grid method [35]. The inclusions were designed to have a fixed unit cell spacing of $8 \mathrm{~mm}$ (Fig. 2). Within this fixed spacing, the inclusion radius was varied $(0.25-1.5 \mathrm{~mm})$ to change the effective inclusion concentration while maintaining a standardized design. In order to minimize transient effects and ensure that characterization occurred well into the crack propagation regime of the experiment, all of the composite designs investigated in this study had their inclusions confined to a single region of interest, as shown as a box in Fig. 3(a). This region was chosen to be 25-30 $\mathrm{mm}$ away from the leading edge of the sample to allow sufficient space for crack nucleation. The region was kept small enough to allow for macroscopic evaluation of toughness using a far-field $J$-integral in the homogeneous domain surrounding the inclusions.

3.3 Mechanical Characterization by Surfing Load and $J$-Integral Analysis. Based on the mathematically described surfing boundary condition, a surfing load fixture, shown in Fig. 4, was devised to produce stable macroscopic crack growth in heterogeneous specimens containing arbitrary composite structures. Unlike traditional testing methods, which are designed to apply loads at a fixed position of the specimen during the entire test, here displacement is continuously adjusted along the specimen as the crack extends. More critically, the resultant load is always a local tensile opening load that does not rely on either a bending moment or specimen geometry to maintain stability. This implies that the crack can change path within the specimen without creating distortion in the far-field loading.

The experimental surfing boundary condition is applied through a combination of rollers and rails. The rail assembly, shown in Fig. 4(a), consists of two parallel sections of rail connected by a diverging zone. The size and angle of the diverging section are chosen such that, so long as the sample is sufficiently plate-like, a tensile opening displacement is prescribed which is large enough to propagate a crack in the specimen, but sufficiently small to prevent instability during crack growth. The rail itself is assembled with two spacers (A2 and A3 in Fig. 4(a)), the thickness of which is chosen so that out of plane motion and specimen drift are minimized. The specimen, shown schematically in Fig. 3(a), is installed between the two rails (Fig. 4(b)) and held in place with rods and rollers (parts $\mathrm{C} 1$ and $\mathrm{C} 3$ in Fig. $4(b)$ ). The rollers are intended to move smoothly along the rail such that the width of the rail along the divergent section controls the applied opening displacement by controlling the exact position of the rods. The rails are connected to a linear stage controlled by a vertical stepping motor (Fig. 4(c)). As a result, when the linear stage is moved downward, the rollers slide along the rail. With this arrangement, the applied displacement field is controlled through the outer profile of the rail, and the rate of crack growth is controlled by the velocity at which the linear actuators move the rail. Further details regarding this assembly can be found in Ref. [36].

In order to measure the driving force on the crack, this experimental configuration can be integrated with non-contact full-field measurement methods such as the digital image correlation and the grid method. In our work, we employed the grid method, which is reviewed in greater detail in Ref. [37]. In this study, a powder-based grid method was used to measure the displacement and strain field, as this approach minimizes the impact of the mounted grid on the resultant toughness measurement, especially for materials with low toughness or high compliance [35]. The measured full-field displacement and the computed strain fields were then used in the area $J$-integral formulation to calculate the macroscopic driving force on the crack [32]. Because toughening due to compliance contrast happens at discrete locations within the composite structure, namely at the interfaces between regions of different compliance, it is not reasonable to treat composites with macroscopic compliance contrast as effectively homogeneous

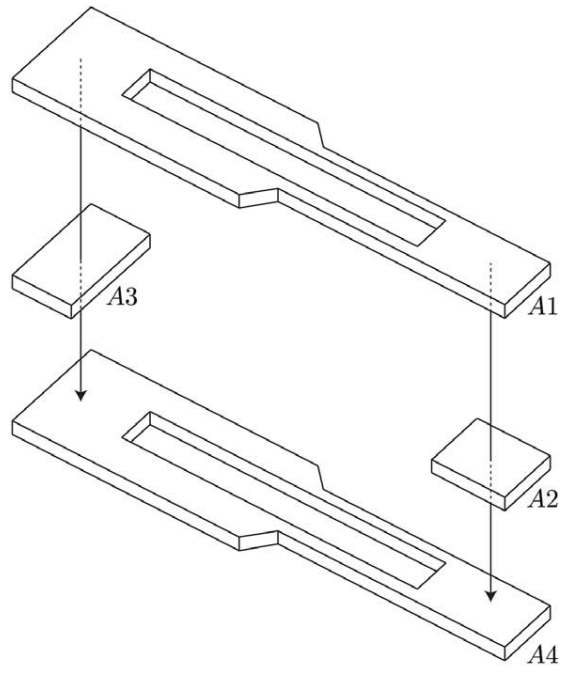

(a)

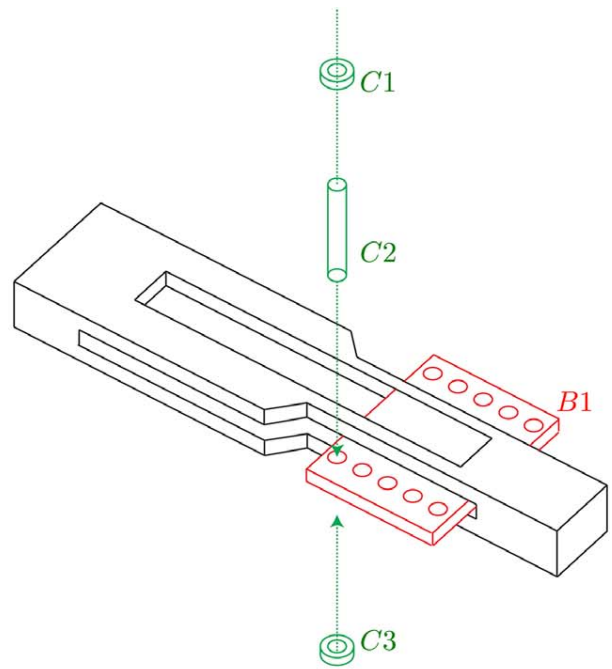

(b)

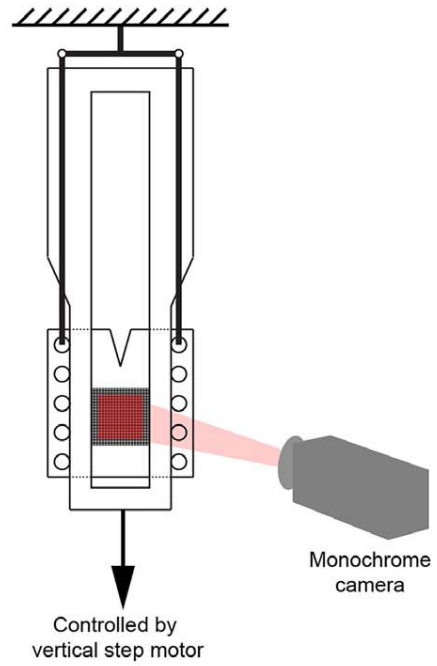

(c)

Fig. 4 Schematic description of the experimental configuration: (a) rail assembly, (b) rail and specimen assembly, and (c) overall configuration 
materials when evaluating failure behavior. For this reason, when evaluating toughness, only macroscopic $J$-integrals encompassing the entirety of the composite structure were considered.

\section{Guiding and Trapping Cracks}

To explore the influence of inclusions on guiding and trapping cracks, simulations were done with normalized matrix and inclusion properties. For these simulations, inclusion size and spacing were scaled relative to the domain size, rather than in absolute dimensional units. We start with an example where toughness is uniform, but the effective Young's modulus of the inclusions is onetenth of that of the matrix. Figure 5 shows the situation where the radius of the inclusions is 0.95 . As the initial crack is driven to the right, the inhomogeneous elastic field due to the compliant inclusion attracts the crack toward the inclusion. However, this heterogeneity is not strong enough and the crack never reaches the inclusion. Therefore, the crack goes on a zig-zag path between the inclusions. We also see that the normalized $J$-integral increases with a peak value of 1.348 as the crack passes near the inclusions; consequently, the effective toughness of this medium is 1.348: note that this is strictly higher than the uniform point-wise values. This example shows that compliant inclusions attract the crack and that can lead to toughening.

We now slightly increase the size of the inclusions to a radius of 1.0, and the results are shown in Fig. 6. The crack is attracted by the compliant inclusions and initially goes very close to the inclusions, almost but not quite reaching the inclusion. However, as the propagation proceeds, the crack deflects sufficiently to touch the inclusion. The crack then propagates along the interface deflecting further, but has a difficult time reentering the matrix. It is trapped and the $J$-integral rises steadily until it reaches a critical value of
1.857 before it breaks free of the interface, reenters the matrix, and tries to recenter. However, it gets attracted and trapped by the next inclusion and the cycle repeats. This example shows not only that compliant inclusions attract the crack, but cracks can get trapped at the interface between the stiff matrix and compliant inclusions leading to additional toughening.

This trapping and toughening increases further when the radius of the inclusions increases to 1.5 as shown in Fig. 7. Now the crack is attracted to the compliant inclusion very quickly and is pinned at the interface for longer periods leading to an even larger effective toughness of 2.009 .

Finally, we close with a comment about the intermediate case of radius 1 shown in Fig. 6. This example also shows that a small perturbation to the crack path while it is in the matrix can lead to a change in its further propagation and required driving force. However, once it gets trapped, small perturbations do not have a significant effect. So, a proper definition of effective toughness should be the smallest possible value over all possible starting points. This is the reason that our simulations always start the crack at the midpoint between two rows of inclusions: all other starting points lead to an equal or lower value of effective toughness.

\section{Improved Properties}

The simulations in Figs. 5-7 demonstrate that compliant inclusions can be used to guide and trap cracks, and this can lead to improved toughness. However, the inclusions also reduce the stiffness of the composite media, so it is important to optimize the combination of properties. Figure 8(a) shows the results of simulations with varying radii of inclusion. The moduli of inclusions and matrix are 0.1 and 1 , respectively, and the toughness is uniformly

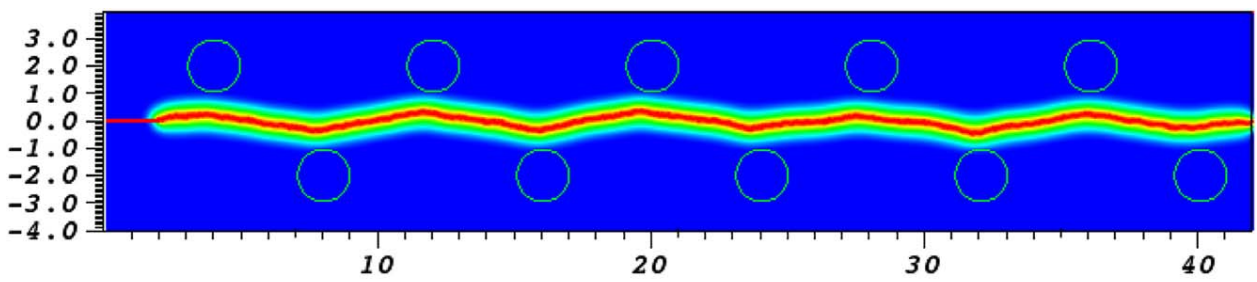

(a)

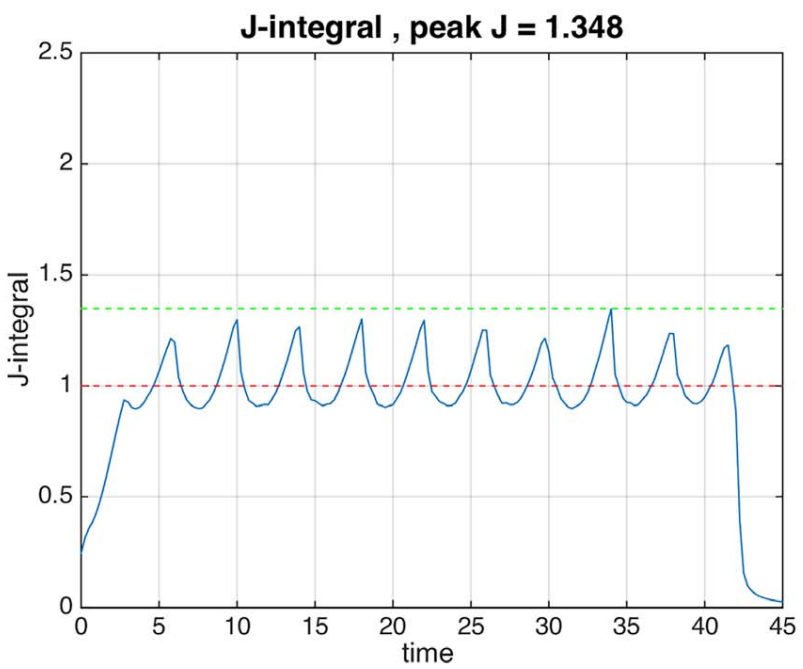

(b)

Fig. 5 (a) Simulation of material with a staggered array of inclusions where the radius of inclusion is 0.95 (inclusion size and spacing chosen relative to domain size) and (b) normalized macroscopic $J$-integral showing the toughness increase due to interaction between cracks and compliant inclusions (the value of the critical energy release rate $\left(J_{c}\right)$ for the base materials is normalized to 1 , and the effective toughness is the maximum value of the normalized $J$-integral) 


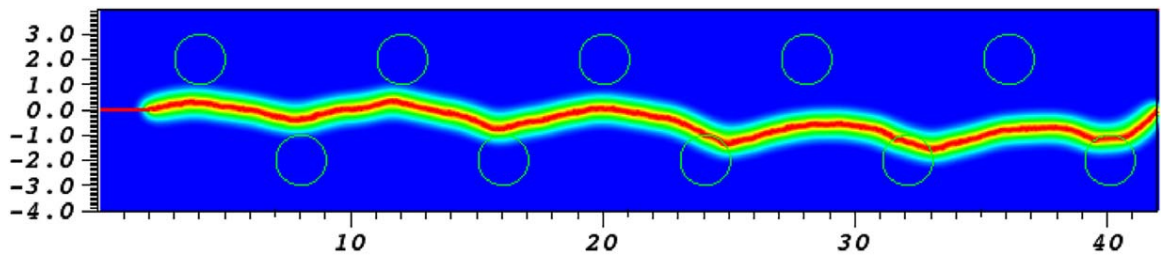

(a)

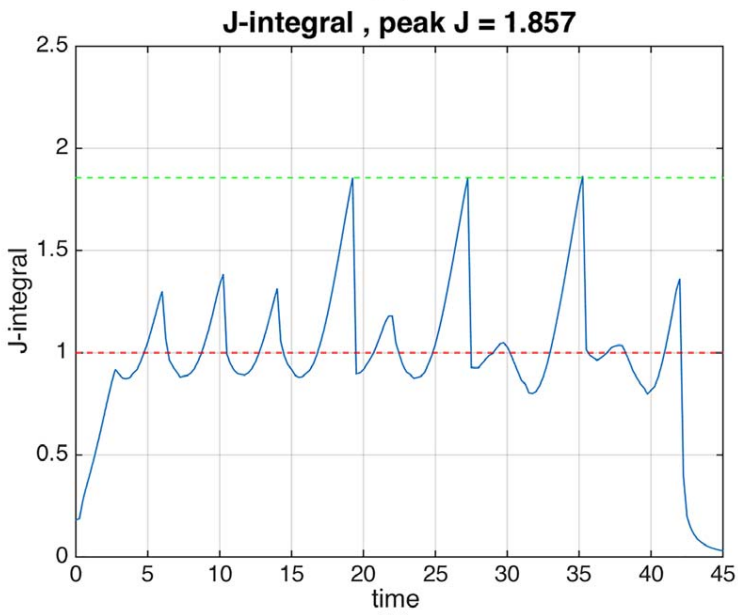

(b)

Fig. 6 (a) Simulation of material with a staggered array of inclusions where the radius of inclusion is 1.0 (inclusion size and spacing chosen relative to domain size) and (b) normalized macroscopic $J$-integral showing the toughness increase due to interaction between cracks and compliant inclusions (the value of the critical energy release rate $\left(J_{c}\right)$ for the base materials is normalized to 1 , and the effective toughness is the maximum value of the normalized $J$-integral)

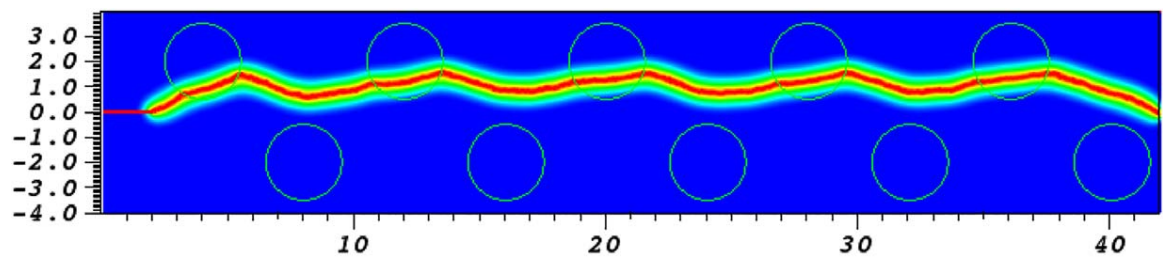

(a)

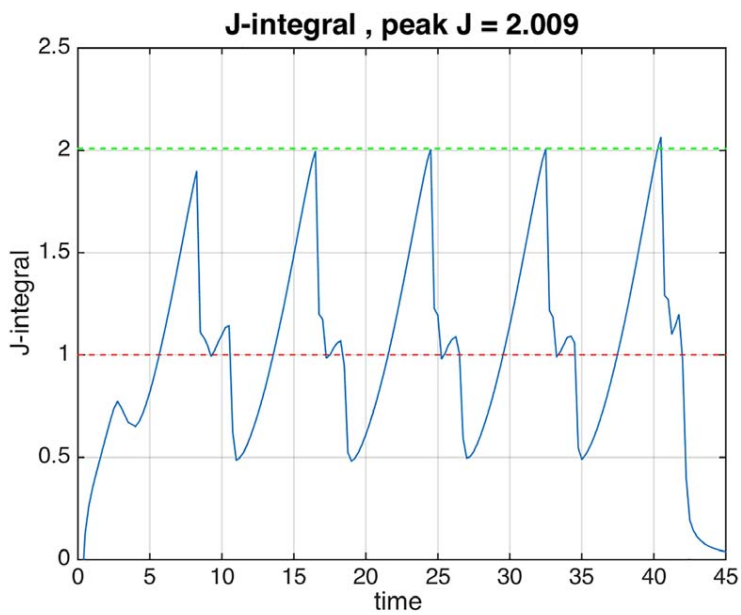

(b)

Fig. 7 (a) Simulation of material with a staggered array of inclusions where the radius of inclusion is 1.5 (inclusion size and spacing chosen relative to domain size) and (b) normalized macroscopic J-integral showing the toughness increase due to interaction between cracks and compliant inclusions (the value of the critical energy release rate $\left(J_{c}\right)$ for the base materials is normalized to 1 , and the effective toughness is the maximum value of the normalized $J$-integral) 


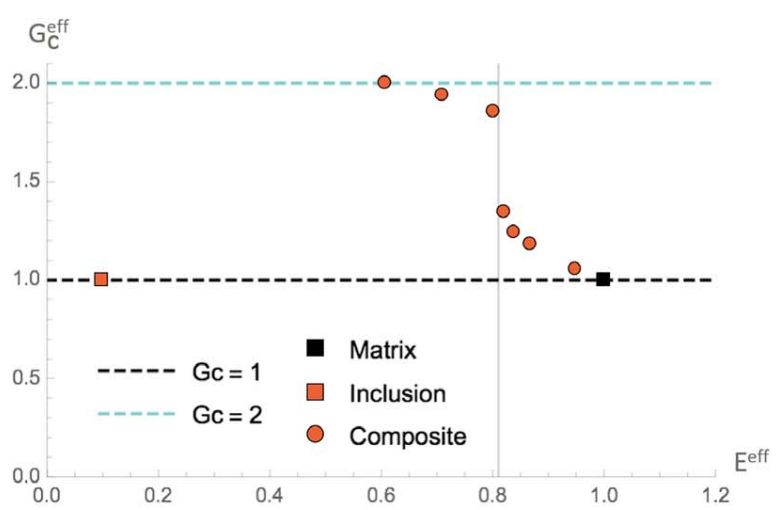

(a)

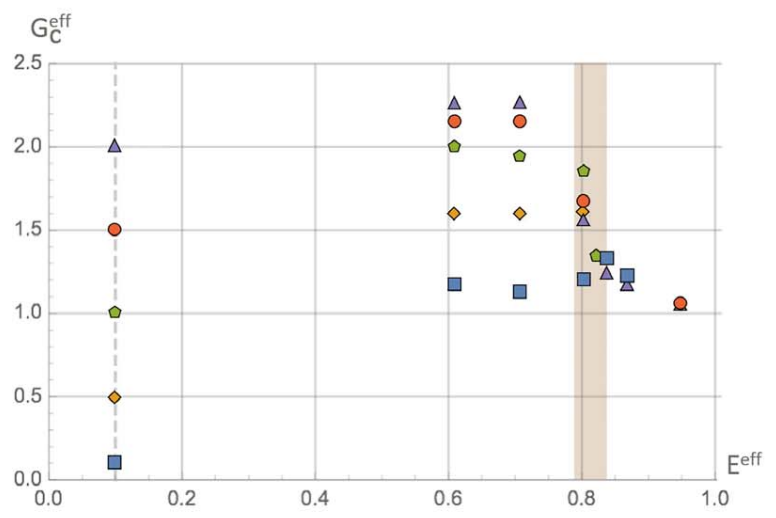

(c)

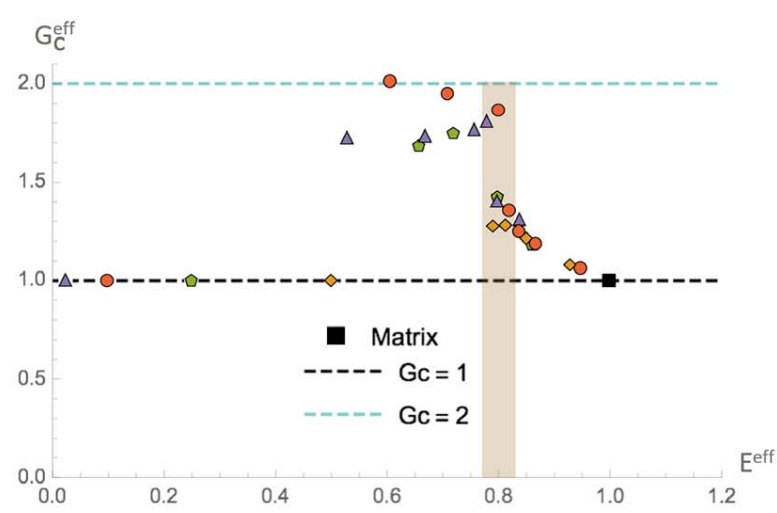

(b)

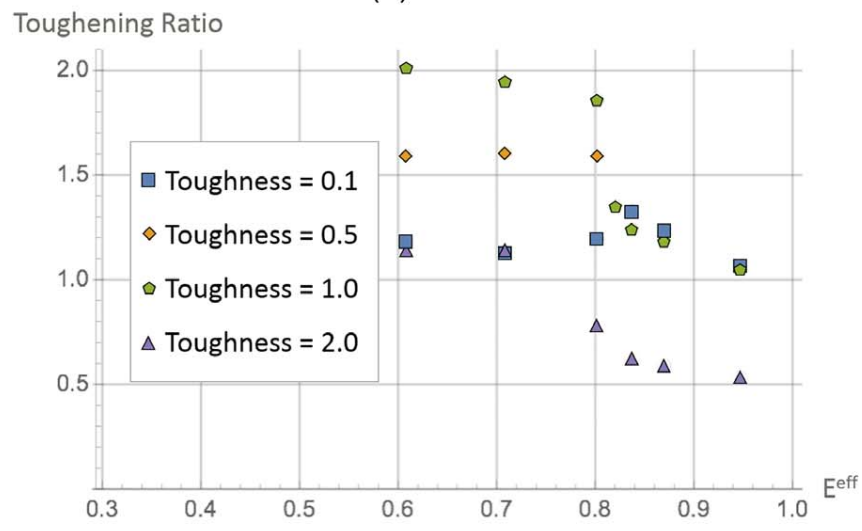

(d)

Fig. 8 Effective toughness and toughening ratio as a function of the effective elastic modulus of the composite material with square array of inclusions. (a) Toughness response for a single system with differing inclusion radii, with square markers indicating matrix and inclusion properties. (b) Toughness response for several systems of differing inclusion stiffness, with points along the toughness $=1$ line indicating inclusion and matrix properties. Highlighted region indicates a transition zone where inclusions begin trapping cracks. (c) Toughness response for several systems of inclusions with fixed stiffness of 0.1 and differing toughness, with inclusion properties for each system shown along the dotted line. Highlighted region indicates transition zone where inclusions begin trapping cracks. (d) Toughening ratio for inclusions of fixed stiffness 0.1 and differing toughness values, where the ratio is the magnitude of the composite toughening normalized to the toughness of the inclusion.

1 (dashed line). The square dot is the matrix material and the square dot is the inclusion material. The circular dots are the effective toughness of the heterogeneous materials for various radii, and consequently, varying effective moduli. The results show that when the inclusion radius is increased, the effective stiffness decreases and the effective toughness increases. Moreover, there is clearly a transition zone (the vertical line) across which the effective toughness has a dramatic jump with little change in effective modulus. This coincides exactly with the transition from attraction to trapping (recall Figs. 5 and 6). The peak value of the toughness is 2.09 (Fig. 7).

These simulations are repeated for various values of the elastic moduli of the inclusion (while holding the elastic modulus of the matrix fixed at 1.0 and the toughness uniform at 1.0 as before). The results are consolidated in Fig. 8(c) where each geometric shape represents a set of simulations for various inclusion radii having a particular value of inclusion modulus. Note that each set shows a transition behavior from a small effective toughness to large effective toughness (shaded region), and the transition is associated with the change from deflection to trapping. We also observe that in each case, the toughness eventually falls after peaking with a further increase in inclusion radius. This is associated with the fact that the inclusions become sufficiently large that cracks do not have to deviate too much to reach consecutive inclusions. Thus, the trapped cracks have a large mode-I driving force, and find it easier to penetrate into the matrix.
Finally, we study the relation between the inclusion toughness and the effective toughness. In this study, we fix the inclusion stiffness at 0.1 and vary the toughness of the inclusions. We use inclusion toughnesses of $0.1,0.5,1.0,1.5$, and 2.0 and plot the effective toughness as a function of effective modulus in Fig. 8(c). The inclusion is shown as the dots on vertical dashed line in the figure. We see that there is a transition zone around an effective stiffness of 0.8 . The effective toughness is largely independent for radii that are small enough that the cracks do not touch the inclusion, as shown by effective stiffness values to the right of the transition zone. However, the inclusion toughness can significantly influence the effective behavior left of the transition zone. Furthermore, higher inclusion toughness leads to higher effective toughness for larger inclusions (left of the transition zone). To understand the true enhancement of the toughness, we define

$$
\begin{aligned}
& \text { Toughening ratio } \\
& \quad=\frac{\text { Effective toughness }}{\text { Max(matrix toughness, inclusion toughness) }}
\end{aligned}
$$

and plot the toughening ratio in Fig. $8(d)$ for these simulations. We see that there is an optimal toughness ratio between the matrix and inclusions $(=1)$ to maximize the toughening ratio. 


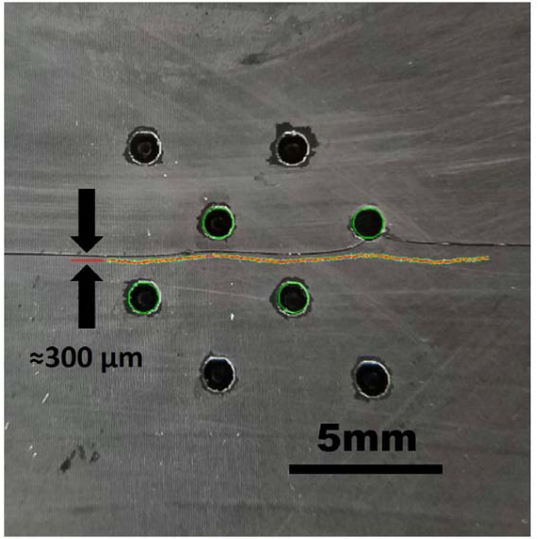

(a)

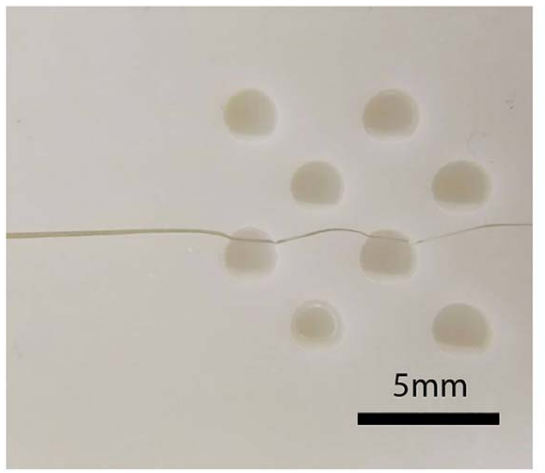

(c)

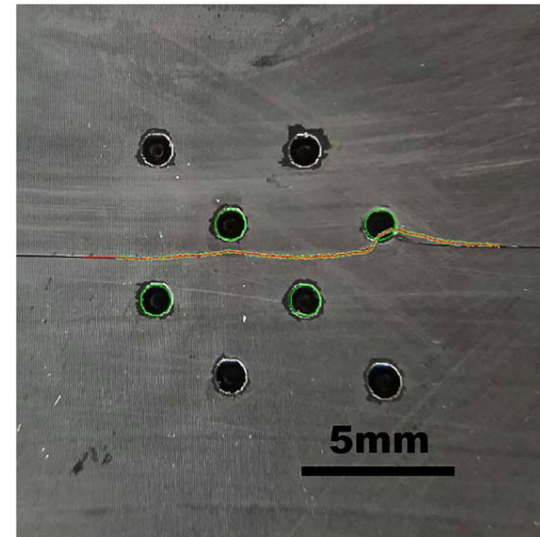

(b)

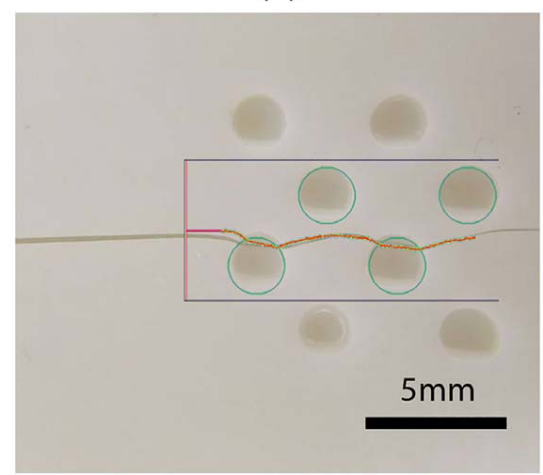

$(d)$

Fig. 9 Comparison of theory and experiment: guiding and trapping cracks. (a) Comparison between experiment and simulation for inclusions of radius $R=0.5 \mathrm{~mm}$ showing a difference in behavior due to difference in crack position. (b) Comparison of experiment and simulation for $R=0.5 \mathrm{~mm}$ after alignment of crack position in simulation. (c) Experiment showing crack interaction with inclusions at a larger radius $R=1 \mathrm{~mm}$. (d) Comparison of experiment and simulation for $R=1 \mathrm{~mm}$.

\section{Comparison Between Theory and Experiment}

We conducted a series of experiments to examine whether compliant inclusions do indeed attract and trap cracks as suggested by the computations above. Figure 9 shows the results of one such experiment conducted on a 3D-printed specimen, the design of which is shown in Fig. 3. Figure $9(a)$ shows the experimentally observed crack path while Figs. $9(b)$ and $9(c)$ show a comparison between the observed and simulated path. These simulations were conducted with the modulus and toughness of the inclusions to taken to be 0.3 times that of the matrix, in accordance with the experimental parameters. We see reasonably good agreement. The first inclusion attracts the crack slightly but the crack path recovers to nearly its original trajectory. The second inclusion then further attracts it, and in fact, traps it. While there is a good overall agreement, there are two issues to note. First, the observed and computed crack paths deviate as they negotiate the inclusion. Second, Figs. $9(a)$ and $9(b)$ highlight the sensitivity of the initial crack position: if the computational crack is off-set by as little as $300 \mu \mathrm{m}$, the crack is no longer trapped.

We now turn to the effective toughness, and the results of the computation and experiment are shown in Fig. 10. As anticipated the toughness of the composite material increases with the presence of the inclusion. However, there are two major differences between simulation and experiment. First, the transition associated with trapping occurs much earlier than predicted. As noted for Figs. 9(a) and $9(b)$, small changes in the location of the initial crack can lead to different propagation scenarios. As evidenced, small misalignment of the specimen leads to a modified crack path which leads to trapping. Thus, the regime where the crack meanders between the inclusions is difficult to achieve in the experiment.
Second, the effective toughness is higher than that predicted for the composite medium. We hypothesize that this is a result of the difference between the way inclusions are modeled in the computations and the way they are introduced in the experiment. In our 2D computations, we assume plane stress conditions, in which both matrix and inclusion are assumed to be of equal thickness. In

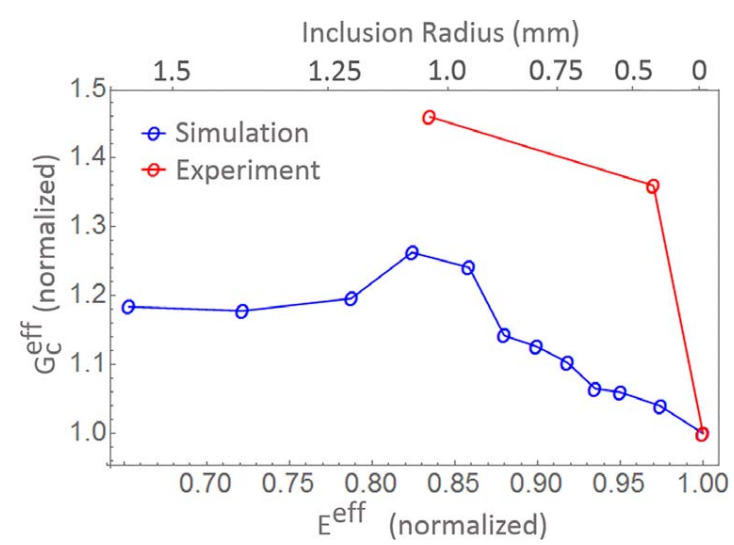

Fig. 10 Comparison of effective stiffness and effective energy release rate (normalized to the matrix material) for composite structures with staggered patterns off isotropic circular inclusions made by varying sample thickness. Comparison shows both expected toughness from simulation as well as toughness measured in experiment. 


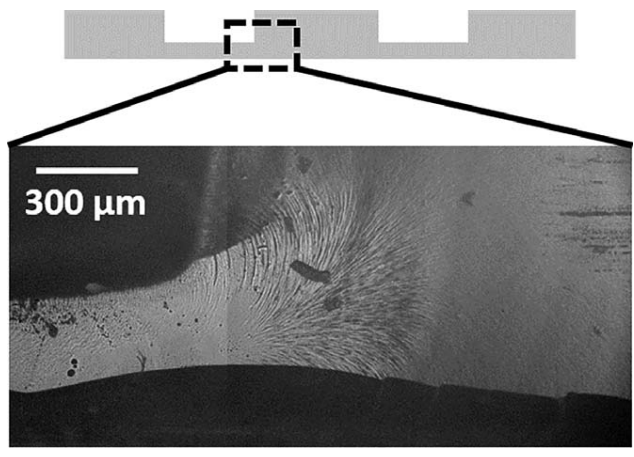

Fig. 11 Optical micrograph showing the unintended geometrical toughening effect in the $E^{\text {eff }}=0.83$ sample. Because elastic contrast was introduced through-thickness variation, the crack was forced to bow outward around the thickness change when exiting the inclusion, creating additional toughening.

contrast, in the experiments, inclusions are introduced by changing material thickness. As a crack negotiates the transition from low stiffness (thin) to high stiffness (thick) sections, the threedimensional aspect of crack propagation must be considered. This is clear in the fractograph in Fig. 11 where the crack has propagated from the inclusion (thin region) to the matrix (thick region) and shows clear evidence of crack bowing. This introduces additional trapping at the interface and is responsible for the higher effective toughness. We further note that the difference in the crack path in Fig. $9(b)$ is also likely the result of this difference between computational assumption and experimental design.

Finally, we note that there are no experimental data for inclusions of larger radii $\left(E^{E f f}<0.83\right.$. As inclusion size increases, the amount of matrix material decreases, such that cracks regularly jump across multiple inclusions.

\section{Conclusion}

We examined the role of microstructure in determining the overall or effective toughness of a heterogeneous material in a model composite. The model system consisting of elastic heterogeneities with a stiff matrix and compliant inclusions made of the same material was investigated using both numerical simulations and experiments. Phase-field numerical simulations were performed of a crack initiating and propagating in a heterogeneous material of a square face-centered array of circular compliant inclusions in a stiff matrix. The simulations showed that substantial toughening could be achieved based purely on the elastic heterogeneity (difference in elastic modulus) between the matrix and the inclusions. It was observed that the crack either meanders or is attracted and trapped by the inclusions depending on the spacing of the inclusions relative to the size of the heterogeneity for a given stiffness. Brittle composite specimens were 3D printed with inclusions of higher compliance introduced through a change in thickness, which resulted in elastic heterogeneity without introducing heterogeneity in toughness. Fracture experiments were performed using a specially designed fixture to maintain stable crack growth, and the $J$-integral was evaluated using the full-field displacement measurements of displacements using the grid method. Cracks were attracted and trapped in the compliant inclusions, which was in accordance with the predictions of the simulations. However, the measured energy release rates were higher than the predicted values, which is attributed to the $3 \mathrm{D}$ effects associated with crack bowing while transitioning from thin to thick sections in the 3D printed specimens. This study provides guidelines for optimal elastic contrast and inclusion geometry for significantly enhancing the toughness without much compromise in the effective elastic modulus of heterogeneous materials.

\section{Acknowledgment}

This work draws from the doctoral theses of Chun-Jen Hsueh and Neal Brodnik at the California Institute of Technology, both of whom contributed equally to this work. We gratefully acknowledge the financial support of the US National Science Foundation (Grant No. DMS-1535083 and 1535076) under the Designing Materials to Revolutionize and Engineer our Future (DMREF) Program.

\section{References}

[1] Evans, A. G., and Faber, K. T., 1981, "Toughening of Ceramics by Circumferential Microcracking," J. Am. Ceram. Soc., 64(7), pp. 394-398.

[2] Faber, K. T., and Evans, A. G., 1983, "Crack Deflection Processes-I. Theory," Acta. Metall., 31(4), pp. 565-576.

[3] Faber, K. T., and Evans, A. G., 1983, "Crack Deflection Processes-II. Experiment," Acta. Metall., 31(4), pp. 577-584.

[4] Suresh, S., 1985, "Fatigue Crack Deflection and Fracture Surface Contact: Micromechanical Models," Metall. Trans. A, 16(2), pp. 249-260.

[5] Gao, H., and Rice, J. R., 1989, "A First-order Perturbation Analysis of Crack Trapping by Arrays of Obstacles,” ASME Trans. J. Appl. Mech., 56(4), pp. 828-836.

[6] Hutchinson, J. W., and Suo, Z., 1992, "Mixed Mode Cracking in Layered Materials," Adv. Appl. Mech., 29(63), p. 191

[7] Cox, B., and Yang, Q., 2006, "In Quest of Virtual Tests for Structural Composites," Science, 314(5802), pp. 1102-1107.

[8] Menig, R., Meyers, M. H., Meyers, M. A., and Vecchio, K. S., 2000, "Quasi-Static and Dynamic Mechanical Response of Haliotis Rufescens (abalone) Shells," Acta Mater., 48(9), pp. 2383-2398.

[9] Evans, A. G., Suo, Z., Wang, R. Z., Aksay, I. A., He, M. Y., and Hutchinson, J. W., 2001, "Model for the Robust Mechanical Behavior of Nacre," J. Mater. Res., 16(9), pp. 2475-2484.

[10] Kumar, P., Nukala, V. V., and Šimunović, S., 2005, "Statistical Physics Models for Nacre Fracture Simulation,” Phys. Rev. E - Stat., Nonlinear, Soft Matter Phys., 72(4), pp. 1-9.

[11] Dirr, N., and Yip, N. K., 2006, "Pinning and De-Pinning Phenomena in Front Propagation in Heterogeneous Media," Interfaces Free Boundaries, 8(1), pp. 79-109.

[12] Barthelat, F., and Espinosa, H. D., 2007, "An Experimental Investigation of Deformation and Fracture of Nacre-Mother of Pearl," Exp. Mech., 47(3), pp. 311-324.

[13] Begley, M. R., Philips, N. R., Compton, B. G., Wilbrink, D. V., Ritchie, R. O., and Utz, M., 2012, "Micromechanical Models to Guide the Development of Synthetic 'brick and Mortar' Composites," J. Mech. Phys. Solids, 60(8), pp. $1545-1560$.

[14] Bouchaud, E., 1997, "Scaling Properties of Cracks," J. Phys.: Condens. Matter, 9(21), pp. 4319-4344.

[15] Ramanathan, S., Ertaş, D., and Fisher, D. S., 1997, "Quasistatic Crack Propagation in Heterogeneous Media,” Phys. Rev. Lett., 79(5), pp. 873-876.

[16] Bonamy, D., Ponson, L., Prades, S., Bouchaud, E., and Guillot, C., 2006, "Scaling Exponents for Fracture Surfaces in Homogeneous Glass and Glassy Ceramics," Phys. Rev. Lett., 97(13), pp. 1-4.

[17] Bonamy, D., Santucci, S., and Ponson, L., 2008, "Crackling Dynamics in Material Failure As the Signature of a Self-organized Dynamic Phase Transition," Phys. Rev. Lett., 101(4), pp. 1-4.

[18] Ponson, L., and Bonamy, D., 2010, "Crack Propagation in Brittle Heterogeneous Solids: Material Disorder and Crack Dynamics," Int. J. Fracture, 162(1-2), pp. 21-31.

[19] Francfort, G. A., and Marigo, J.-J., 1998, "Revisiting Brittle Fracture As An Energy Minimization Problem," J. Mech. Phys. Solids, 46(8), pp. 1319-1342.

[20] Bourdin, B., Francfort, G. A., and Marigo, J.-J., 2000, "Numerical Experiments in Revisited Brittle Fracture," J. Mech. Phys. Solids, 48(4), pp. 797-826.

[21] Bourdin, B., 2007, "Numerical Implementation of a Variational Formulation of Quasi-Static Brittle Fracture," Interfaces Free Bound., 9(3), pp. 411-430.

[22] Bourdin, B., Francfort, G. A., and Marigo, J.-J., 2008, "The Variational Approach to Fracture," J. Elasticity, 91(1-3), pp. 1-148.

[23] Bourdin, B., Marigo, J.-J., Maurini, C., and Sicsic, P., 2014, "Morphogenesis and Propagation of Complex Cracks Induced by Thermal Shocks," Phys. Rev. Lett., 112(1), p. 014301.

[24] Pham, K., Amor, H., Marigo, J.-J., and Maurini, C., 2011, "Gradient Damage Models and Their Use to Approximate Brittle Fracture," Int. J. Damage Mech., 20(4, SI), pp. 618-652.

[25] Pham, K., Marigo, J.-J., and Maurini, C., 2011, "The Issues of the Uniqueness and the Stability of the Homogeneous Response in Uniaxial Tests with Gradient Damage Models," J. Mech. Phys. Solids, 59(6), pp. 1163-1190.

[26] Tanné, E., Li, T., Bourdin, B., Marigo, J-J., and Maurini, C., 2018, "Crack Nucleation in Variational Phase-field Models of Brittle Fracture," J. Mech. Phys. Solids, 110, pp. 80-99.

[27] Bourdin, B., 2019, "mef90/vDef: Variational Models of Defect Mechanics," https://github.com/bourdin/mef90

[28] Hossain, M. Z., Hsueh, C. J., Bourdin, B., and Bhattacharya, K., 2014, "Effective Toughness of Heterogeneous Media," J. Mech. Phys. Solids, 71(1), pp. 15-32.

[29] Moulinec, H., and Suquet, P., 1995, "A FFT-Based Numerical Method for Computing the Mechanical Properties of Composites From Images of Their Microstructures," IUTAM Symposium on Microstructure-Property Interactions in Composite Materials, Aalborg, Denmark, Aug. 22-25, 1994, Springer, New York, pp. 235-246. 
[30] Moulinec, H., and Suquet, P., 1998, "A Numerical Method for Computing the Overall Response of Nonlinear Composites With Complex Microstructure," Comput. Meth. Appl. Mech. Eng., 157(1-2), pp. 69-94.

[31] Michel, J. C., Moulinec, H., and Suquet, P., 2001, "A Computational Scheme for Linear and Non-linear Composites with Arbitrary Phase Contrast," Int. J. Numerical Methods Eng., 52(1-2), pp. 139-160.

[32] Hsueh, C.-J., 2017, "Effective Toughness of Heterogeneous Materials," $\mathrm{PhD}$ thesis, California Institute of Technology, Pasadena, CA.

[33] Wang, N., and Xia, S., 2017, "Cohesive Fracture of Elastically Heterogeneous Materials: An Integrative Modeling and Experimental Study," J. Mech. Phys. Solids, 98, pp. 87-105.
[34] AutoDesk, 2017, “Autodesk Standard Clear PR48 Formulation,” https://cdn2. hubspot.net/hubfs/1545937/Autodesk_Standard_Clear_PR48_Formulation.pdf? - his

[35] Brodnik, N. R., Hsueh, C. J., and Johnson, M. T., 2018, "A Nonconstraining Templated Powder Grid for Measurement of Strain," Strain, 54, p. e12273.

[36] Brodnik, N. R., 2019, "Fracture and Toughening of Brittle Structures With Designed Anisotropy," PhD thesis, California Institute of Technology, Pasadena, CA

[37] Grédiac, M., Sur, F., and Blaysat, B., 2016, "The Grid Method for In-plane Displacement and Strain Measurement: A Review and Analysis," Strain, 52(3), pp. 205-243. 